\title{
La calidad de los servicios universitarios: reflexiones a partir del estudio de casos en el contexto latinoamericano
}

Agustín Mejías Acosta* Mauricio Valle Barra** Arturo Vega Robles***

\section{RESUMEN}

El objetivo de esta investigación fue identificar los factores que determinan la calidad de los servicios académicos en instituciones universitarias, a partir de tres estudios de casos en el contexto latinoamericano (Venezuela, México y Chile). Se usó el modelo de encuesta SERVQUALing, y previa demostración de la adecuación muestral, se empleó la técnica estadística multivariante de Análisis de Factores, donde se identificaron las dimensiones que subyacen la calidad de los servicios, según las percepciones de los estudiantes, destacándose la Seguridad, la Capacidad de Respuesta y la Empatía, como elementos clave y comunes. La encuesta usada resultó fiable y válida, según lo evidencian las pruebas estadísticas realizadas (significativas al $5 \%$ ). Los resultados confirman el carácter dinámico y multidimensional de la calidad de los servicios académicos, lo que permite presentar algunas reflexiones para el debate.

Palabras clave: calidad de servicio, universidad, análisis de factores, SERVQUALing, Ingeniería Industrial

THE QUALITY OF UNIVERSITY SERVICES: REFLECTIONS FROM A CASE STUDY IN THE LATIN AMERICAN CONTEXT

\section{ABSTRACT}

The objective of this research is to identify factors that determine the quality of academic services in universities, from three case studies in the context of Latin America ( Venezuela, Mexico and Chile). Model survey SERVQUALing was used, and upon proof of sampling adequacy, the multivariate statistical technique of Factor Analysis, where the dimensions underlying quality of services as perceived by students were identified was used highlighting Safety, Responsiveness and Empathy as a key and common elements. The survey used was reliable and valid, as evidenced by the statistical tests performed ( significant at $5 \%$ ). The results confirm the dynamic and multidimensional nature of quality of academic services, allowing present some thoughts for discussion.

Keywords: Service quality, University, factor analysis, SERVQUALing , Industrial Engineering

\section{INTRODUCCIÓN}

El concepto de calidad en el servicio ha sido definido e interpretado de diferentes formas a lo largo del tiempo; es el tema más importante, más extensamente estudiado y de mayor debate en el campo de los servicios (Wankhade y Dabade, 2010), de hecho, como lo señalan Trujillo, Carrete, Vera y García (2011), evaluar la calidad en los servicios es un proceso altamente complejo que funciona en varios niveles de abstracción.

La calidad del servicio se ha convertido en un requisito para todas las empresas y organizaciones exitosas para mantener la competitividad (Grönroos, 1988). La clave para el logro de la calidad en el servicio, implica atender los procesos desde su conceptualización hasta su aseguramiento y garantía, logrando que realmente satisfagan a los usuarios, no solo en sus necesidades sino en sus deseos y expectativas que ellos tienen dentro del contexto de la eficacia, consiguiendo que los clientes no solamente sean leales a la organización sino también, comenten su satisfacción con las personas con las cuales se relacionan diariamente (Vargas y Aldana, 2006).

La conceptualización y medición de la calidad de servicio ha sido un tema difícil de comprender como consecuencia de la intangibilidad de los servicios, y los problemas asociados con su producción y recepción simultanea (Lloréns, 1996); y aun cuando la comunidad científica no se pone de acuerdo en este debate (Ramezani, Baharun y Feiz, 2012), la literatura sobre el tema evidencia principalmente dos corrientes de investigación, la Nórdica liderada por Grönroos y la Americana liderada por Parasuraman, Zeithaml y Berry (Brady y Cronin, 2001; Duque, 2005; Mejías y Maneiro, 2007). Sin embargo, la mayoría de las experiencias coinciden en conceptualizar sus experiencias a partir del enfoque basado en percepciones (de Grönroos) y la medición con base en los trabajos de Parasuraman et al.

La calidad de servicio es definida formalmente por Parasuraman et al. (1985) como el grado y dirección de la diferencia entre las expectativas y las percepciones que tiene el cliente de la calidad de servicio prestado. El enfoque sobre la calidad percibida en los servicios, como lo señala Grönroos (1994), quién introdujera

\footnotetext{
* Ingeniero Industrial, M. Sc. Dr., Docente Investigador Universidad de Carabobo, Venezuela E-mail: aamejiasa@gmail.com

** Ingeniero Industrial, M. Sc., Dr., Docente Investigador Universidad de Valparaiso, Chile. E-mail: mauricio.valle@uv.cl

*** Ingeniero Industrial, M. Sc., Docente Investigador Universidad de Sonora, México. E-mail: avega@caborca.uson.mx
} 
este enfoque en 1982, parece ser la base de la mayor parte de las investigaciones que se siguen realizando sobre la calidad de los servicios, a pesar de los intentos de estudiarla desde otra perspectiva; sin embargo, el SERVQUAL de parasuraman et al. $(1988,1991)$ es el modelo de encuesta más usado y aceptado para la medición de la calidad de los servicios (Lloréns, 1996; Salvador, 2010; Hoffman y Bateson, 2008); pero también el más criticado y de mayor controversia (Cronin y Taylor, 1994; Asubonteg et al., 1996, Buttle, 1996, entre otros).

Como lo señalan Arambewela y Hall (2006), la fuerza impulsora de la globalización es la competencia y el mercado de la educación internacional se ha vuelto muy competitivo con diferentes estrategias de marketing implementadas por las instituciones educativas para atraer al creciente número de estudiantes que buscan educación superior. Esta alta competitividad en el sector de la educación superior, el aumento en el número de universidades y la cada vez mayor exigencia de los alumnos, ha llevado a las universidades a centrar cada vez más sus esfuerzos en ofrecerles un mejor servicio con el objetivo de satisfacer sus necesidades (Torres y Araya, 2010); a esto se agrega la creciente competencia por los escasos recursos con lo que cuentan las instituciones de educación superior, que en la mayoría de las veces vienen del sector gubernamental.

Shekarchizadeh, Rasli y Hon (2011), apoyándose en otros autores, aseguran que existe un cuerpo sustancial de evidencia en la literatura de educación superior que sugiere que el SERVQUAL es eficaz para medir la calidad del servicio en el entorno de la educación superior. En este contexto son muchas las iniciativas emprendidas cuyo soporte o medio de búsqueda de información y datos ha sido el SERVQUAL; así se destacan los trabajos de Mejías, Maneiro y Reyes (2006) quienes evalúan la calidad de los servicios en la educación mexicana; Maneiro, Mejías, Romero y Zerpa (2008), quienes plantean una evaluación de la calidad de servicio, usando una versión del SERVQUAL previamente validada por Mejías (2005a), a fin de obtener información para determinar el perfil del ingeniero industrial y su posicionamiento en el mercado laboral venezolano; De Oliveira, y Ferreira (2009) quienes realizan un estudio en un programa de ingeniería en la Universidad de San Pablo en Brasil; Clemenza, Azuaje y Araujo (2010), quienes determinan la calidad de servicio de las instituciones privadas de educación superior en una región venezolana; por su parte, Vergara y Quezada (2011), analizan la calidad en el servicio y satisfacción de los estudiantes de una universidad colombiana; y
Almeida et al. (2012), quienes evalúan la calidad de los servicios en un programa de Ingeniería de producción en la Universidad Federal de Paraná, Brasil; entre muchos otros.

En el sub sector de estudios para postgraduados, son resaltantes los trabajos de Mejías (2005b) quién propone un modelo fundamentado en el SERVQUAL para medir la Calidad del Servicio en los Estudios Universitarios de Postgrado; Arambewela y Hall (2006), quienes examinan la relación entre los constructos del SERVQUAL y el país de origen y la satisfacción de estudiantes internacionales de postgrado asiáticos que estudian en universidades australianas; Shekarchizadeh, Rasli y Hon (2011) quienes reportan aplicaciones en Malasia; entre otros.

En este trabajo y con base en los estudios de Grönroos y Parasuraman et al, se define calidad de servicios como la percepción que tienen los estudiantes sobre el grado en el que se le han cumplido sus necesidades; y como escala para su medición la versión SERVQUALing (Mejías, 2005; Mejías y Maneiro, 2007) que toma en cuenta solamente las percepciones del SERVQUAL (Parasuraman et al., 1988, 1991).

Previa introducción en el tema de calidad de servicios en el contexto de educación superior, este artículo se estructura presentando la metodología usada para seguir la investigación, con base principalmente en la técnica estadística de "Análisis de Factores"; luego se presentan y discuten los resultados, comparándolos entre los casos en estudio, así como con los estudios previos en el área. Finalmente se presentan, a manera de conclusiones, algunas reflexiones para el debate.

\section{METODOLOGÍA}

El presente trabajo corresponde a una investigación transversal, de tipo exploratorio (Hernández, Fernández y Baptista, 2010); fue realizada como parte de la línea de investigación de "Modelado y análisis estadístico de datos en gestión de la calidad", en el segundo semestre de 2012, mediante estudios de tres casos en Escuelas de Ingeniería Industrial de universidades en Venezuela (Caso 1), México (Caso 2) y Chile (Caso 3), que forman parte del grupo de investigación y de la Red Internacional de Investigadores en Ingeniería Industrial <Redl4>. Se empleó el modelo de encuesta SERVQUALing (Mejías y Maneiro, 2007), instrumento usado para medir las percepciones de los estudiantes de la carrera de Ingeniería Industrial, que es una versión del SERVQUAL de Parasuraman et al. 
(1988, 1991). Para la selección de los tamaños de muestra, se usó el muestreo no probabilístico (Grande y Abascal, 2005; dada las condiciones de la población en estudio, tomando en cuenta el carácter exploratorio de la investigación. Los tamaños de muestras fueron $\mathrm{n}_{1}=197, \mathrm{n}_{2}=100 \mathrm{y}$ $\mathrm{n}_{3}=85$ para los casos de Venezuela, México y Chile; 1,2 y 3 , respectivamente.

Para la identificación de los factores que subyacen a la calidad de los servicios académicos prestados se usó la técnica de análisis de datos multivariante de "Análisis de Factores", previa demostración de la adecuación de la calidad de los datos para realizar dicho análisis. El análisis de factores es una técnica de reducción de datos que sirve para encontrar grupos homogéneos de variables a partir de un conjunto numeroso de variables (Pardo y Ruiz, 2002). El procesamiento de los datos se realizó con ayuda de la hoja de cálculo Excel de Microsoft $\circledast$ y del software IBM SPSS $®$.

\section{RESULTADOS Y DISCUSIÓN}

La escala usada en los tres casos en estudios fue la misma, SERVQUALing presentada en Cuadro 1. Esta escala fue diseñada para medir la calidad de servicio percibida en educación superior (Mejías 2005; Mejías y Maneiro, 2007), y tiene como base las cinco (05) dimensiones y veintidós (22) variables propuestas por Parasuraman et al. (1988, 1991).

\section{Cuadro 1. Dimensiones de la calidad de servicio en educación superior}

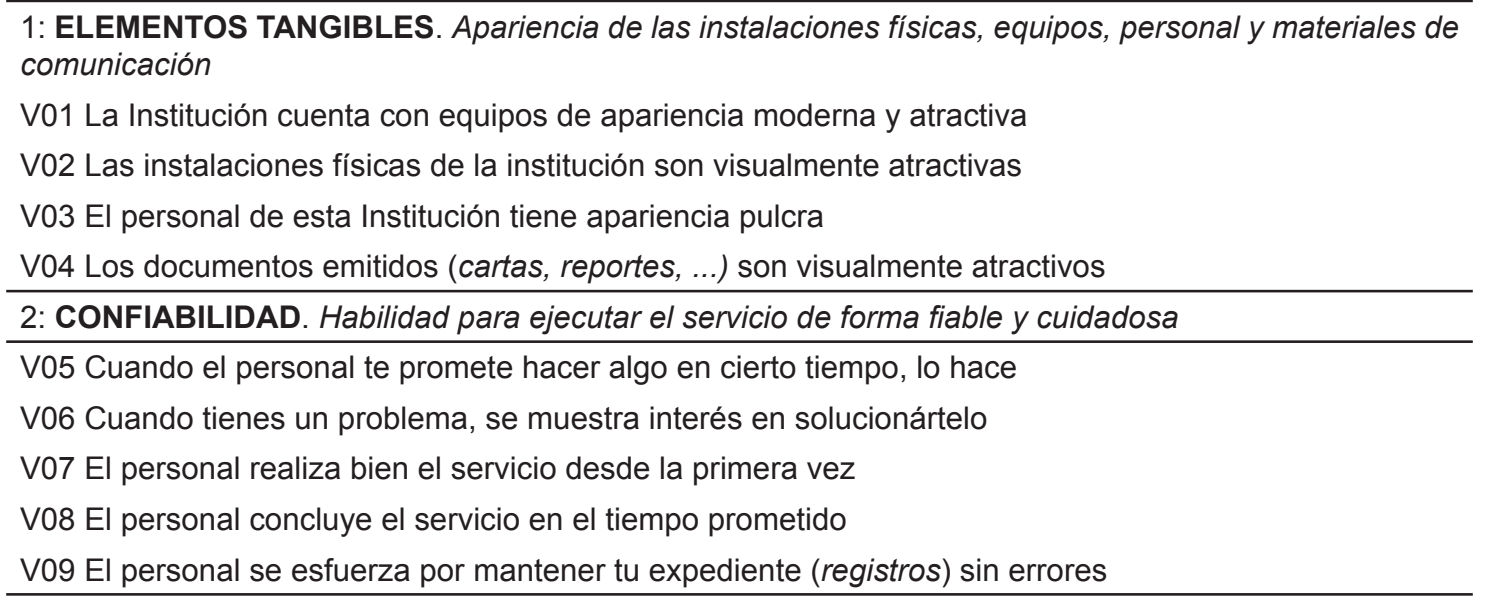

3: CAPACIDAD DE RESPUESTA. Disposición y voluntad del personal para ayudar al estudiante y proporcionar el servicio

V10 El personal te comunica cuando concluirá el servicio prestado

V11 En esta Institución se te ofrece un servicio puntual

V12 El personal de esta institución siempre está dispuesto a ayudarte

V13 El personal de esta institución siempre está disponible para atenderte

4: SEGURIDAD. Conocimientos y atención mostrados por el personal y sus habilidades para inspirar credibilidad y confianza

V14 El comportamiento del personal te inspira confianza

V15 Te sientes seguro en tus trámites realizados en esta Institución

V16 El personal que presta servicios en esta institución es amable contigo

$\mathrm{V} 17 \mathrm{El}$ personal tiene conocimientos suficientes para responderte

5: EMPATíA. Atención individualizada que se ofrece a los estudiantes

V18 El personal de esta institución te da una atención individualizada

V19 Esta Institución tiene horarios de trabajo convenientes sus estudiantes

V20 En esta Institución se te ofrece una atención personalizada

V21 El personal se preocupa por los mejores intereses de sus estudiantes

V22 En esta institución se comprenden tus necesidades específicas

Fuente: (Adaptado de Mejías y Maneiro, 2007) 
Una vez obtenida la opinión de los estudiantes, los datos fueron registrados en una matriz que serviría como base para el Análisis de Factores; esta técnica, se inicia con la evaluación de la matriz de los coeficientes de correlaciones entre cada par de variables; para que el uso de la técnica sea pertinente, es conveniente que esta matriz de correlaciones contenga grupos de variables que se correlaciones fuertemente entre sí (Pardo y Ruiz, 2002), ya que se espera determinar los factores subyacentes que explican tales correlaciones (Tapia, 2007).

Dos de los indicadores que se usan para evidenciar la conveniencia de esta matriz, y por ende la adecuación de los datos para el uso del Análisis de Factores, son el Determinante de dicha matriz y el Índice KMO. El determinante de la matriz es un indicador del grado de intercorrelación; si es muy pequeño, es decir cercano a cero (0), indica que el grado de intercorrelación es muy grande, condición inicial que debe cumplir el análisis (Pérez, 2005; Vicente y Oliva y Manera, 2003). Para los casos de estudio, los valores del determinante de la matriz de coeficientes de correlación son Det $_{1}=1 \mathrm{E}-4$, Det $_{2}=9,52 \mathrm{E} 6$ y Det $_{3}=5,4 \mathrm{E}-5$, evidenciando un alto grado de correlación entre las variables.

EI KMO (Kaiser-Meyer-Olkin) es una medida basada en los coeficientes de correlación observados de cada par de variables y en sus coeficientes de correlación parcial. En el caso de que exista adecuación de los datos a un modelo factorial, el KMO será próximo a la unidad (1); mientras más cerca esté de 1 , mayor es la adecuación de los datos, considerándose valores aceptables los mayores a 0,5 , y excelentes los mayores a 0,9 (Pérez, 2007). Para los casos en estudio, se reportan valores mayores a $0,850\left(\mathrm{KMO}_{1}=0,879\right.$; $\mathrm{KMO}_{2}=0,895$ y $\mathrm{KMO}_{3}=0,839$ ), por lo que se puede considerar que los datos son adecuados para el análisis de factores, tomando en cuenta otros autores además de Pérez (2007), quienes también consideran que entre 0,8 y 0,9 los valores del KMO son muy buenos (Vicente y Oliva y Manera, 2003). Como se reportan valores de los determinantes muy pequeños y valores de la medida $\mathrm{KMO}$ mayores a 0,8 , se podría esperar un buen ajuste de los datos para aplicar la técnica de análisis de factores.

Para extraer los factores, se usó el método de componentes principales, usando como criterio los autovalores mayores que 1 . Para el caso 1 de la Universidad Venezolana, se obtuvieron 4 factores que logran explicar el $56,5 \%$ de la varianza total, con comunalidades mayores a 0,500; para el caso 2 , se tienen 4 factores que representan el $62,02 \%$ de la varianza total y comunalidades mayores a 0,490 ; y para el caso 3, 4 factores que logran explicar el $66,2 \%$ de la varianza total y comunalidades mayores a 0,550 .

Para lograr una mejor interpretación de la estructura de los datos analizados, se realiza una rotación mediante el método Varimax, el cual, en términos generales, minimiza el número de variables que tienen cargas altas en un factor, permitiendo identificar los factores representados en los cuadros 2, 4 y 6 para cada caso, respectivamente.

Como parte del análisis de factores, y con base en los criterios de parsimonia de Thurstone (1947, citados por Kerlinger y Lee, 2002) que establecen que se debería tomar la explicación más simple de aquellas que sean coherentes con los datos, ciertas variables fueron excluidas de los modelos representados en los cuadros 2, 4 y 6; así mismo, algunas variables que presentaban correlaciones no significativas con el resto, tampoco fueron incluidas en el modelo en el proceso previo de demostración de la adecuación muestral.

De la matriz rotada representada en el Cuadro 2 se pueden identificar cuatro (4) factores latentes que agrupan diecisiete (17) de las veintidós (22) variables originales del modelo SERVQUALing. En el cuadro 3, una descripción de las dimensiones identificadas.

Cuadro 2. Matriz de componentes rotados para el caso 1 (Venezuela)

\begin{tabular}{lllll}
\hline & \multicolumn{4}{l}{ Componente } \\
\cline { 2 - 5 } V07 & 1 & 2 & 3 & 4 \\
V22 &, 730 & & & \\
V11 &, 714 & & & \\
V13 &, 691 & & & \\
V06 &, 691 & & & \\
V01 & & & & \\
V08 & &, 722 & & \\
V10 & &, 641 & & \\
V04 & &, 569 & & \\
V05 & &, 549 & & \\
V03 & & &, 733 & \\
V17 & & &, 622 & \\
V12 & & &, 557 & \\
V20 & & &, 537 & \\
V15 & & &, 525 & \\
V09 & & & &, 741 \\
V16 & & & &, 724 \\
\hline
\end{tabular}

Fuente: Elaboración propia. 
En el primer factor, se destacan los aspectos relacionados con la Confiabilidad como la buena realización del servicio desde la primera vez y el interés en la solución de problemas; sin embargo, otras variables como puntualidad y disponibilidad de capacidad de respuesta son incluidas en este primer factor.

En el segundo factor, se agrupan variables relacionadas con la promesa y el cumplimiento de la realización del servicio (confiabilidad), su comunicación oportuna (capacidad de respuesta) y la apariencia de los equipos y documentos (tangibles), en una dimensión que se podría identificar como efectividad de servicio. En el tercer factor, se destacan aspectos relacionados con la Seguridad en el servicio ofrecido, donde generalmente se destacan los conocimientos y la capacidad para inspirar confianza, pero que en este caso incluye la apariencia y el comportamiento del personal como punto clave.

Un cuarto factor representa la Amabilidad que incluye el esfuerzo y amabilidad del personal. Una descripción de las dimensiones y sus variables (o ítems) para este caso se presenta en el Cuadro 3.

Cuadro 3: Dimensiones de la calidad de servicio en educación superior.

Caso 1 Universidad pública venezolana

\begin{tabular}{l}
\hline 1: CAPACIDAD DE RESPUESTA. Disposición del personal para ayudar y proporcionar el servicio \\
\hline V07 EI personal realiza bien el servicio desde la primera vez \\
V22 En esta escuela se comprenden tus necesidades específicas \\
V11 En esta escuela se te ofrece un servicio puntual \\
V13 EI personal de esta escuela siempre está disponible para atenderte \\
V06 Cuando tienes un problema, se muestra interés en solucionártelo \\
\hline 2: EFECTIVIDAD DE SERVICIO. Efectividad, en la oferta, comunicación y conclusión del servicio, así \\
como la apariencia de los equipos y documentos emitidos \\
\hline V01 La escuela cuenta con equipos de apariencia moderna y atractiva \\
V08 EI personal concluye el servicio en el tiempo prometido \\
V10 EI personal te comunica cuando concluirá el servicio prestado \\
V05 Cuando el personal te promete hacer algo en cierto tiempo, lo hace \\
V04 Los documentos emitidos son visualmente atractivos \\
\hline 3: SEGURIDAD. Conocimientos, apariencia y habilidad del personal para inspirar credibilidad y confianza \\
\hline V03 El personal de esta escuela tiene apariencia pulcra \\
V17 EI personal tiene conocimientos suficientes para responderte \\
V12 EI personal de esta institución siempre está dispuesto a ayudarte \\
V20 Esta institución te ofrece una atención personalizada \\
V15 Te sientes seguro en tus trámites realizados en esta institución \\
\hline 4: AMABILIDAD. Cortesía y esfuerzo personal \\
\hline V09 EI personal se esfuerza por mantener tus registros sin errores \\
V16 EI personal que presta servicios es siempre amable contigo \\
\hline
\end{tabular}

Fuente: Elaboración propia.

En el Cuadro 4 se reporta la matriz rotada obtenida de los datos del segundo caso de estudio correspondiente a una Universidad Mexicana. Para la representación de la estructura de los datos obtenidos en este caso, se identifica, al igual que en el caso venezolano, los aspectos relacionados con la disposición del personal para ayudar al estudiante y ofrecer un buen servicio; en este caso vale la pena destacar el aspecto clave que representan los conocimientos del personal que generalmente están en el factor Seguridad.

En el segundo factor se agrupan variables que caracterizan la Empatía del personal, a saber la atención individualizada y personalizada, 
completando este factor los documentos, los horarios y el servicio puntual. En el factor 3 , donde se agrupan los elementos tangibles, se destaca, además de la apariencia pulcra del personal la capacidad de este para inspirar confianza a sus estudiantes. Los aspectos relacionados con la
Seguridad y Oportunidad se agrupan, para este caso, en el cuarto factor.

En el Cuadro 5 se resumen las dimensiones de la calidad de los servicios académicos para el caso de la Universidad Mexicana.

Cuadro 4. Matriz de componentes rotados para el caso 2. Universidad Mexicana

\begin{tabular}{lcccc}
\hline & \multicolumn{5}{c}{ Componente } \\
\cline { 2 - 5 } V17 & 1 & 2 & 3 & 4 \\
V13 &, 750 & & & \\
V21 &, 735 & & & \\
V07 &, 690 & & & \\
V12 &, 614 & & & \\
V10 &, 600 & & & \\
V06 & &, 707 & & \\
V19 & &, 691 & & \\
V04 & &, 691 & & \\
V11 & &, 653 & & \\
V20 & &, 622 & & \\
V18 & &, 600 & & \\
V01 & & &, 704 & \\
V02 & & &, 624 & \\
V03 & & &, 586 & \\
V14 & & & & \\
V05 & & & & \\
V15 & & & & \\
V16 & & & & \\
V09 & & & & \\
\hline
\end{tabular}

Fuente: Elaboración propia.

Cuadro 5. Dimensiones de la calidad de servicio en educación superior. Caso 1 Universidad Mexicana

1: CAPACIDAD DE RESPUESTA. Disposición del personal para ayudar y proporcionar el servicio

V17 El personal tiene conocimientos suficientes para responderte

V13 El personal siempre está disponible para atenderte

V21 El personal se preocupa por los mejores intereses de sus estudiantes

V07 El personal realiza bien el servicio desde la primera vez

V12 El personal de esta escuela siempre está dispuesto a ayudarte

V10 El personal te comunica cuando concluirá el servicio prestado

2: EMPATIA. Atención individualizada y personalizada que ofrece la Institución a sus estudiantes

V06 Cuando tienes un problema, se muestra interés en solucionártelo

V19 La escuela tiene horarios de trabajo convenientes 
V04 Los documentos emitidos son visualmente atractivos

V11 En esta escuela se te ofrece un servicio puntual

V20 Esta Institución te ofrece una atención personalizada

V18 El personal de esta escuela te da una atención individualizada

3: ELEMENTOS TANGIBLES. Apariencia de las instalaciones físicas, equipos, personal y materiales de comunicación

V01 La escuela cuenta con equipos de apariencia moderna y atractiva

V02 Las instalaciones físicas de éste campus son visualmente atractivas

V03 El personal de esta escuela tiene apariencia pulcra

V14 El comportamiento del personal de esta escuela te inspira confianza

4: SEGURIDAD y OPORTUNIDAD. Habilidad para realizar el servicio prometido de forma segura y oportuna

V05 Cuando el personal te promete hacer algo en cierto tiempo, lo hace

V15 Te sientes seguro en tus trámites realizados en esta Escuela

V16 El personal de esta Institución es siempre amable contigo

V09 El personal se esfuerza por mantener tus registros sin errores

Fuente: Elaboración propia.

Para el caso de la Universidad Chilena, el tercero en estudio, en el primer factor se reúnen variables relacionadas con la seguridad y la confianza que inspira el personal, incluyéndose además, la preocupación, el interés y la disposición a ayudar. En el segundo factor, se incluyen aspectos de confiabilidad y cuidado en el cumplimiento del servicio. En el tercer factor se reúnen aspectos de empatía y amabilidad mostrados por el personal; finalmente, el cuarto factor, reúne indiscutiblemente los aspectos relacionados con los elementos tangibles.

En la matriz rotada para el caso 3 de la Universidad Chilena, se evidencian variables agrupadas en cuatro (4) factores.
Cuadro 6. Matriz de componentes rotados para el caso 3. Universidad Chilena.

\begin{tabular}{lllll}
\hline & \multicolumn{4}{l}{ Componente } \\
\cline { 2 - 5 } V15 & 1 & 2 & 3 & 4 \\
V06 &, 818 & & & \\
V07 &, 809 & & & \\
V20 &, 804 & & & \\
V13 &, 727 & & & \\
V09 &, 536 & & & \\
V05 & &, 788 & & \\
V12 & &, 717 & & \\
V11 & &, 705 & & \\
V03 & &, 546 & & \\
V16 & &, 528 & & \\
V14 & & &, 738 & \\
V18 & & &, 662 & \\
V02 & & &, 612 & \\
V21 & & &, 601 & \\
V23 & & &, 521 & \\
V04 & & & &, 893 \\
V08 & & & & \\
\hline
\end{tabular}

Fuente: Elaboración propia. 
Cuadro 7: Dimensiones de la calidad de servicio en educación superior.

Caso 3 Universidad Chilena

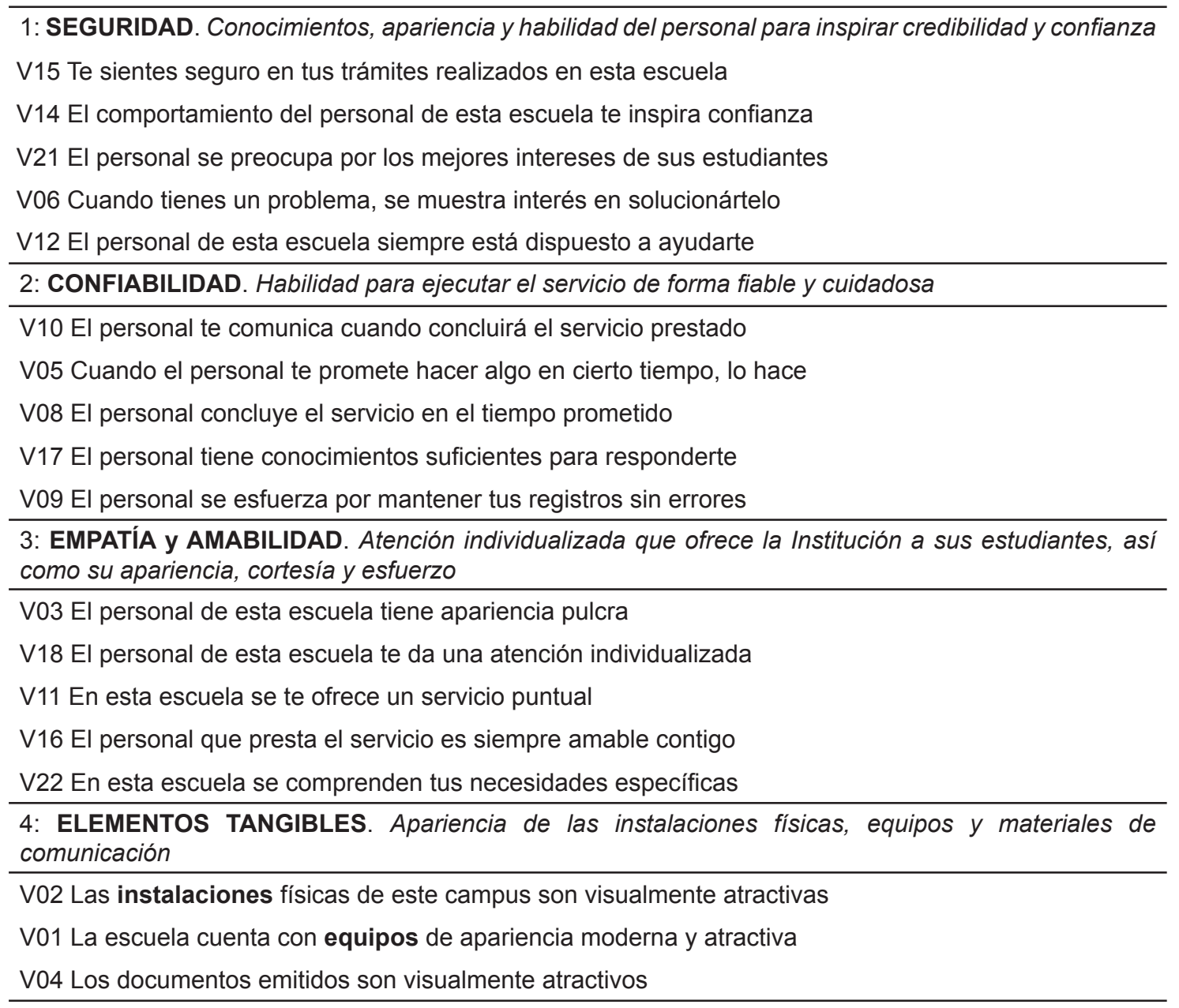

Fuente: Elaboración propia.

\section{VALIDEZ Y FIABILIDAD DE LA ESCALA}

\subsection{Determinación de la validez}

La validez de las mediciones de los constructos o variables utilizados en marketing es una condición indispensable para el desarrollo y contraste de teorías científicas (Martínez y Martínez, 2009). Este proceso de validación aglutina un conjunto de estudios encaminados a proveer a las puntuaciones de una encuesta de una interpretación teórica coherente con relación a un contexto de uso bien limitado (Oliden, 2003).

Según Hernández et al. (2010), la validez de una escala es el grado en que dicha escala realmente mide la variable que pretende medir; mientras que la AERA (1999) la define como el grado en que la evidencia y la teoría apoyan las interpretaciones dadas a los resultados de las encuestas realizadas con base en el uso propuesto.

La validez se refiere a la credibilidad y fundamento que tienen las interpretaciones derivadas de los resultados de la aplicación de una encuesta, así como su relevancia y significación con respecto al contexto en el cual se desean plantear conclusiones. Para determinar la validez de la escala SERVQUALing se presentan tres enfoques, tratando de resumir la extensa bibliografía del tema: validez de contenido, validez de constructo y validez de criterio.

La validez de contenido es el grado en que un instrumento refleja el dominio especifico de contenido de lo que se medir (Hernández et al., 2010); es importante ya que hace referencia a la adecuada selección de las medidas de la variable 
de interés (Martínez y Martínez, 2009). Las veintidós variables que integran la escala SERVQUALing son una adaptación del conocido modelo SERVQUAL, en la cual solo se toma en cuenta las percepciones de los usuarios y no las diferencias entre estas percepciones y las expectativas previas. El modelo SERVQUAL (Parasuraman et al., 1988, 1991) es el más usado en la actualidad para medir la calidad de servicio, tal como se evidencia en la revisión de literatura presentada en la sección de Introducción; por su parte la escala SERVQUALing ha sido aplicada en muchos casos para la evaluación de la calidad de los servicios académicos, por lo que se puede considerar que ésta escala presenta validez de contenido.

La validez de criterio o validez pragmática, establece la validez de un instrumento de medición comparándola con algún criterio externo (Hernández et al., 2010); si se fija un criterio en el presente se conoce como concurrente, y si se fija el criterio en el futuro, se le denomina predictiva.

En los casos en estudio, para determinar la validez concurrente, se clasificaron los encuestados en dos categorías, una formada por puntuaciones menores a la media y otros con puntuaciones mayores, con baja percepción y con alta percepción respectivamente. Tomando como referencia una pregunta adicional presentada en el instrumento relacionada con la percepción general de la calidad de servicio ofrecida, y con base en una prueba estadística no paramétrica $U$ de Mann-Whitney (Montogomery y Runger), se encontró que existen diferencias significativas (al 5\%) entre las medias de las categorías, lo cual evidencia la validez concurrente de la escala SERVQUALing.

Para evidenciar la validez predictiva, se realizó un análisis de regresión multiple (Montgomery y Runger) tomando como variables independientes los valores promedios de las dimensiones $\left(X_{i}\right)$ y como variable dependiente $(Y)$ una variable adicional a la escala que media la satisfacción de los usuarios sobre los servicios académicos prestados. Los resultados estadísticos $\left(R^{2}\right.$ y pruebas de adecuación del modelo significativos al $5 \%$ ) evidencian la validez predictiva del modelo.

La validez de Constructo (o de concepto), es la principal de los tipos de validez (Pérez, Chacón y Moreno, 2000); se refiere al grado en el que una medición se relaciona de manera consistente con otras mediciones, de acuerdo con hipótesis derivadas teóricamente y que conciernen a los conceptos que se están midiendo (Hernández et al., 2010). La validez de constructo se puede clasificar en convergente y discriminante.
Cuando se busca evidenciar la validez Convergente, se parte del hecho de que la característica en estudio, en este caso la calidad de los servicios académicos, pude ser medida de diferentes formas dando lugar a resultados similares. Para este fin, se realizó un análisis de correlación con los valores promedios de la Escala usada SERVQUALing y una variable adicional presentada a los encuestados que medía la Calidad de Servicios en general. Los resultados (pruebas significativas la $5 \%$ ) confirman la validez convergente de la escala SERVQUALing para los casos en estudio.

Para determinar la validez discriminante (o divergente), se incluyó en la encuesta una variable relacionada con la percepción de la expectativa de nivel de vida al concluir el programa, la cual evidentemente no depende de la calidad de los servicios académicos ofrecidos. Los resultados de las pruebas de correlación realizadas (No significativas al $5 \%$ ) no evidencian correlación entre las variables, por lo cual se pude decir que la escala tiene validez discriminante.

Adicionalmente, se ha usado la técnica de análisis de factores para la identificación de las dimensiones que determinan la calidad de los servicios. Esta técnica es la más usada para determinar la validez de constructo de una escala (Macía, 2010; Pérez, Chacón y Moreno, 2000).

\subsection{Análisis de fiabilidad}

La fiabilidad es la capacidad de la escala (o encuesta) para medir de forma consistente, precisa y sin error la característica que se desea medir (Pardo y Ruiz, 2005). Se dice que un instrumento (o encuesta) es fiable cuando con el mismo se obtienen resultados similares al aplicarlo dos o más veces al mismo grupo de individuos o cuando lo que se aplica o administra son formas alternativas del instrumento (Visauta y Martori, 2003).

La evaluación de la fiabilidad de una escala consiste, precisamente en cuantificar en qué medida son replicables las puntuaciones que ofrece (Prat y Doval, 2005). La consistencia interna es una de las estrategias para evaluar la fiabilidad de una escala, y el coeficiente Alpha de Cronbach es uno de los indicadores más utilizados para este fin (Visauta y Martori, 2003; Prat y Doval, 2005; Quero, 2010).

El Alpha de Cronbach está basado en las correlaciones entre las variables, y se interpreta de manera similar a una probabilidad (entre cero y uno), por lo que mientras más cercanos sean estos valores a uno mejor. 
Los valores alcanzados para el coeficiente Alfa de Cronbach en los casos presentados fueron superiores a 0,650 para cada dimensión, lo cual indica una homogeneidad y equivalencia de respuesta a todos los ítems a la vez y para todos los encuestados.

La alta consistencia interna es un indicador suficientemente válido de que la escala usada mide la calidad de servicio en los casos estudiados, lo que se interpreta como garantía de una alta fiabilidad de la escala.

\section{REFLEXIONES FINALES: A MANERA DE CONCLUSIONES}

La calidad de servicio es un indicador clave para la gestión de las instituciones de educación superior. Su conceptualización y medición en este contexto ha tenido una atención generalizada en los últimos años, tal como lo evidencia la literatura del tema iniciada en los años ochenta por las corrientes de investigación de Grönroos y Parasuraman, enfoques Nórdico y Americano, respectivamente.

Para medir la calidad de los servicios académicos en los casos estudiados correspondientes a tres instituciones de Venezuela, México y Chile, respectivamente, se ha usado la escala SERVQUALing, que es una versión de la escala SERVQUAL de Parasuraman et al.

A pesar de que la estructura subyacente de los datos obtenidos en los casos estudiados no coincide con el modelo teórico SERVQUALing, se identifican dimensiones generales, a saber: Capacidad de respuesta, seguridad y empatía, y destacándose la presencia de variables clave en los procesos educativos como la confianza que genera el personal y los conocimientos.

Así mismo, en comparación con trabajos similares en el contexto de educación superior señaladas en este trabajo, las dimensiones identificadas difieren en cuanto interpretación y enfoque para su análisis, por lo que el trabajo en red podría redundar en el logro de una mayor credibilidad y fundamento de las conclusiones generales, para que tener una destacada relevancia y significación en el contexto latinoamericano.

El estudio de los casos conjuntamente en diferentes instituciones de educación superior, permite abrir un debate sobre las iniciativas de calidad de servicio, por una parte desde el punto de vista de investigación, pero se aprovecharían muchas oportunidades en el componente de gerencia universitaria. Sin duda alguna, es mucho lo que falta para consolidar las corrientes investigativas en el contexto educativo latinoamericano, pero se debe comenzar por compartir los resultados y experiencias.

Con base en los resultados de las pruebas estadísticas realizadas, significativas al 5\%, se puede decir que la escala es válida y fiable para medir la calidad de los servicios académicos universitarios en los casos estudiados.

\section{Agradecimiento}

A la Red Internacional de Investigadores en Ingeniería Industrial <Redl4>, por servir de plataforma para la discusión de iniciativas, y al Consejo de Desarrollo Científico y Humanístico de la Universidad de Carabobo por la subvención al proyecto de investigación. Este trabajo fue presentado en las XIII SEMANA DA ENGENHARIA DE PRODUÇÃO SUL-AMERICANA SEPROSUL 2013, realizadas en la Universidad Federal Do Rio Grande do Sul, Brasil.

\section{REFERENCIAS BIBLIOGRÁFICAS}

[1] Ahmed, M. y Raghunandan, S. (2013). SERVQUAL revisited. A systematic review of literature. International Journal in Multidisciplinary and Academic Research (SSIJMAR), 2 (2), 1-27.

[2] Almeida, C.; Pschera, C; Seleme, R.; Isoldi, S. y Gomes, R. (2012). Percepção da qualidade no ensino superior: aplicação do SERVQUAL no curso de graduação em engenharia de produção da UFPR. Memorias. XL CONGRESSO BRASILEIRO DE EDUCAÇÃO EM ENGENHARIA - COBENGE 2012.

[3] Ruy Gomes Silva Arambewela, R. y Hall, J. (2006). A comparative analysis of international education satisfaction using SERVQUAL. Journal of Services Research, 6(1), 141-163.

[4] Asubonteng, P., Mccleaty, K.J. y Swan, J.E. (1996). SERVQUAL revisited: a critical review of service quality.

[5] Journal of Service Marketing, 10(6), 62-81.

[6] Angell, J., Heffernan, T. y Megicks, P. (2008). Service quality in postgraduate education. Quality Assurance in Education, 16 (3), 236-54.

[7] Brady, M. y Cronin, J. (2001). Some New Thoughts on Conceptualizing Perceived Service Quality: A Hierarchical Approach. The Journal of Marketing, 65 (3), 34-49.

[8] Brochado, A. (2009). Comparing alternative instruments to measure service quality in higher 
education. Quality Assurance in Education, 17 (2), 174-190

[9] Buttle, F. (1996). SERVQUAL: review, critique research agenda. European Journal of Marketing, 30 (1), 8-32.

[10] Clemenza, C., Azuaje, H. y Araujo, R. (2010). Calidad de servicio de las instituciones privadas de educación superior. Omnia, 16 (1), 53-8.

[11] Cronin, J.J. y Taylor, S.A. (1994). SERVPERF versus SERVQUAL: reconciling performance based and perceptions-minus-expectations measurement of service quality. Journal of Marketing, 58 (1), 125-31.

[12] De Oliveira, O. y Ferreira, E. (2009). Adaptation and application of the SERVQUAL scale in higher education Society. 20 th Annual Conference of the Production and Operations Managements, USA, 11-72.

[13] Duque, E. (2005). Revisión del concepto de calidad del servicio y sus modelos de medición. Innovar, 15 (25), 64-80.

[14] Firdaus, A. (2006). The development of HEdPERF: a new measuring instrument of service quality for the higher education sector. International Journal of Consumer Studies, 30 (6), 569-81.

[15] Grande, I. y Abascal, E. (2005). Análisis de encuestas. Madrid: ESIC Editorial.

[16] Gronroos, C. (1988). Service quality: the six criteria of good perceived service. Review of Business, 9 (3), 10-13.

[17] Gronroos, C. (1994). Marketing y gestión de servicios. Madrid: Ediciones Díaz de Santos.

[18] Hernández, R.; Fernández, C. y Baptista, P. (2010). Metodología de la Investigación, 5ta edición. México: Mc Graw Hill.

[19] Hoffman, K. y Bateson, J. (2008). Services marketing: Concepts, strategies \& cases. USA: Cengage learning.

[20] Kerlinger, F. y Lee, H. (2002). Investigación del comportamiento. $4^{\mathrm{a}}$ ed. México: McGraw-Hill.

[21] Macía, F. (2010). Validez de los tests y el análisis factorial: nociones generales. Ciencia \& Trabajo, 12 (35), 276-280.

[22] Martínez, J. y Martínez, L. (2009). Los test estadísticos y la evaluación de escalas; el caso de la invalidez discriminante. Investigaciones Europeas de Dirección y Economía de la Empresa, 15 (2), 15-24.
[23] Maneiro, N.; Mejías, A.; Romero, M. y Zerpa, J. (2008). Evaluación de la calidad de los servicios, una experiencia en la educación superior venezolana. EDUCERE, 12 (43), 797-804.

[24] Mejías, A. (2005a). Validación de un instrumento para medir la calidad de servicio en programas de estudios universitario. Revista Ingeniería Industrial, 27 (2), 20-25.

[25] Mejías, A. (2005b). Modelo para medir la Calidad del Servicio en los Estudios Universitarios de Postgrado. Revista Universidad, Ciencia y Tecnología, 4 (34), 81-85.

[26] Mejías, A. y Maneiro, N. (2007). Medición de la Calidad de Servicio. Serie Cuadernos de Ingeniería Industrial. Valencia: Universidad de Carabobo.

[27] Oliden, P. (2003). Sobre la validez de los tests. Psicothema, 15 82), 315-321.

[28] Parasuraman, A.; Zeithaml, V. y Berry, L. (1985). A Conceptual Model of Service Quality and Its Implications for Future Research. Journal of Marketing, 41-50.

[29] Parasuraman, A.; Zeithaml, V. y Berry, L. (1988). SERVQUAL: A Multiple-Item Scale for Measuring Customer Perceptions of Service Quality. Journal of Retailing, 12-40.

[30] Parasuraman, A.; Zeithaml, V. y Berry, L. (1994). Reassessment of Expectations as a Comparison Standard in Measuring Service Quality: Implications for Future Research. Journal of Marketing, 1, 11-24.

[31] Pardo, A. y Ruiz, M. (2005). Análisis de datos con SPSS. Madrid: McGraw-Hill/Interamericana de España.

[32] Pérez, C. (2005). Métodos Estadísticos Avanzados con SPSS. Madrid: Thomson.

[33] Pérez, C. y Santín, D. (2007). Minería de datos. Madrid: Internacional Thomson Editores Paraninfo.

[34] Pérez, J.; Chacón, S. y Moreno, R. (2000). Validez de constructo: El uso del análisis factorial exploratorio-confirmatorio para obtener evidencias de validez. Psicothema, 12 (2), 442-446.

[35] Prat, R. y Doval, E. (2005). Construcción y análisis estadístico de escalas. En Análisis. Multivariante para las Ciencias Sociales (Comp. Lèvy, J. y Valera, J.). Madrid: Pearson Prentice Hall. 
[36] Quero, M. (2010). Confiabilidad y coeficiente Alpha de Cronbach. TELOS, 12 (2), 248-252.

[37] Ramezani, A.; Baharun, R. y Feiz, S. (2012). A review of service quality models. $2^{\text {nd }}$ International Conference on management Proceeding. Malaysia.

[38] Shekarchizadeh, A., Rasli, A. y Hon-Tat, H. (2011). SERVQUAL in Malaysian universities: perspectives of international students. Business Process Management Journal, 17 (1), 67-81.

[39] Salvador, C. (2010). Quality of University Services: Dimensional Structure of SERVQUAL VS. ESQS

[40] Service Science, 2(3), 167-176.

[41] Tan, K. y Kek, S. (2004). Service Quality in Higher Education Using an Enhanced Servqual Approach. Quality in Higher Education, 10 (1), 17-24.

[42] Thurstone, L. 1947. Multiple-factor analysis. Journal of Consulting Psychology, 11(4), 223-224.
[43] Torres, E. y Araya, L. (2010). Construcción de una escala para medir la calidad del servicio de las universidades: Una Aplicación al Contexto Chileno. Revista de Ciencias Sociales (RCS), 16 (1), 54-67.

[44] Trujillo, A.; Carrete, L.; Vera, J. y García, S. (2011). Servir con calidad en México. México: LID Editorial Mexicana.

[45] Vargas, M. y Aldana, L. (2006). Calidad en el Servicio. Bogotá: ECOE Ediciones.

[46] Vergara, J. y Quesada, V. (2011). Análisis de la calidad en el servicio y satisfacción de los estudiantes de Ciencias Económicas de la Universidad de Cartagena mediante un modelo de ecuaciones estructurales. Revista Electrónica de Investigación Educativa, 13 (1), 108-122.

[47] Visauta, B. y Martori, J. (2003). Análisis estadístico con SPSS para Windows. Madrid: MacGraw-Hill 